\title{
Erratum to: Statistical hadronization and hadronic microcanonical ensemble II
}

F. Becattini ${ }^{a}$, L. Ferroni

Università di Firenze and INFN Sezione di Firenze, Via G. Sansone 1, 50019 Sesto F.no (Firenze), Italy

Received: 26 November 2009 / Published online: 22 January 2010

(C) Springer-Verlag / Società Italiana di Fisica 2010

Erratum to: Eur. Phys. J. C (2004) 38: 225-246

DOI 10.1140/epjc/s2004-02027-8

Equation (34) should read:

$$
\begin{aligned}
P\left(\left\{N_{j}\right\}\right)= & \frac{1}{Z(\mathbf{Q})}\left[\prod_{j} \sum_{\left\{h_{n_{j}}\right\}}(\mp 1)^{N_{j}+H_{j}} \prod_{n_{j}=1}^{N_{j}} \frac{z_{j\left(n_{j}\right)}^{h_{n_{j}}}}{n_{j}^{h_{n_{j}}} h_{n_{j}} !}\right] \\
& \times \delta_{\mathbf{Q}, \sum_{j} N_{j} \mathbf{q}_{j}}
\end{aligned}
$$

Equation (C.7) should read:

$N_{j} ! \mathrm{e}^{-N_{j} \mathbf{i} \mathbf{q}_{j} \cdot \boldsymbol{\phi}} \sum_{\left\{h_{n_{j}}\right\}}(\mp 1)^{N_{j}+\sum_{n_{j}} h_{n_{j}}} \prod_{n_{j}} \frac{z_{j\left(n_{j}\right)}^{h_{n_{j}}}}{n_{j} h_{n_{j}} h_{n_{j}} !}$.
Equation (C.8) should read:

$$
\begin{aligned}
P\left(\left\{N_{j}\right\}\right)= & \frac{1}{Z(\mathbf{Q})}\left[\prod_{j=1}^{K} \sum_{\left\{h_{n_{j}}\right\}}(\mp 1)^{N_{j}+H_{j}} \prod_{n_{j}} \frac{z_{j\left(n_{j}\right)}^{h_{n_{j}}}}{\left.n_{j}^{h_{n_{j}} h_{n_{j}} !}\right]}\right. \\
& \times \delta_{\mathbf{Q}, \sum_{j} N_{j} \mathbf{q}_{j} .}
\end{aligned}
$$

The online version of the original article can be found under doi:10.1140/epjc/s2004-02027-8.

a e-mail: becattini@fi.infn.it 